\title{
QUASI-INVARIANT RADON MEASURES ON GROUPS
}

\author{
CHANDRA GOWRISANKARAN
}

Abstract. Let $G$ be a Hausdorff topological group which is a Baire space. It is proved that if there is a quasi-invariant Radon measure on $G$ then $G$ is locally compact. Examples of non-Baire groups with and without quasi-invariant measures are considered. In particular, it is shown that there is no $\sigma$-finite measure on the Wiener space which preserves sets of measure zero under translation.

Let $G$ be a Hausdorff topological group. A Radon measure $\mu$ on $G$ is said to be left quasi-invariant if for every $\sigma \in G$ the measures $\mu$ and $\mu_{\sigma}$ (the image of the measure $\mu$ under the mapping $x \rightarrow \sigma x$ ) are absolutely continuous relative to each other. This is equivalent to the property that for any compact set $K \subset G, \mu(K)=0$ iff $\mu\left(\sigma^{-1} K\right)=0$. It is a well-known fact that on a locally compact Hausdorff group the left quasi-invariant Radon (q.-i.R.) measures which are also right quasi-invariant are the indefinite integrals, with respect to the left invariant Haar measure, of a positive measurable function $f$ such that $f>0$ except on a locally (Haar) negligible set. However, in case $G$ is not locally compact, there may exist left q.-i.R. measures, even though there does not exist any left invariant Radon measure [3]. All the groups considered below will be Hausdorff. We prove the following:

THEOREM 1. Let $G$ be a topological group which is a Baire space. If there is a nontrivial (丰0) left q.-i.R. measure on $G$ then $G$ is locally compact.

The proof is based on the following lemma and on an idea due to J. C. Oxtoby [5, Theorem 2].

LemMA 1. Let $\mu \neq 0$ be a left q.-i.R. measure on a topological group $G$. Then $\mu(U)>0$ for every nonvoid open set $U \subset G$.

Proof. Suppose on the contrary $\mu(U)=0$ for some nonvoid open set $U$ contained in $G$. Since $\mu$ is quasi-invariant it follows that every point has an open neighbourhood of $\mu$ measure zero. Hence every compact set has $\mu$-measure zero and this in turn implies that $\mu \equiv 0$; a contradiction. The lemma is proved.

Received by the editors February 16, 1972.

AMS 1969 subject classifications. Primary 2875; Secondary 2846.

Key words and phrases. Topological group, quasi-invariant, Radon measure, locally compact, Baire space, vector space, Wiener algebra.

(c) American Mathematical Society 1972 
Proof of THE THEOREM. Since $\mu$ is locally finite and, by the above lemma, we may choose an open set $V$ containing the identity element $e$ such that $0<\mu(V)<+\infty$, we may also assume that $V$ is symmetric. By the inner regularity of $\mu$ we can find a compact set $K \subset V$ such that $\mu(K)>0$ and, if necessary, by replacing $K$ by $K \cup K^{-1}$, which is also contained in $V$, we may assume that $K=K^{-1}$. Let $G_{1}=\bigcup_{n=1}^{\infty} K^{n} ; G_{1}$ is a subgroup of $G$.

Let us suppose that $G$ is not locally compact. Then every compact subset of $G$ has void interior. In particular, $K^{n}$ for each $n$ has void interior. Hence $G_{1}$ is a meagre set. Let, now, $W$ be an open neighbourhood of $e$ such that $W K \subset V$. Let $W^{\prime}$ be a set of representatives from $W$ of the cosets of $W$ by $G_{1}$. Then $F=\bigcup \sigma G_{1}$ for $\sigma \in W^{\prime}$ is a disjoint union of meagre sets and clearly $W \subset F$. Since $G$ is a Baire space so is $W$ and hence $W^{\prime}$ is necessarily an uncountable set. Now, for any $\sigma, \sigma^{\prime} \in W^{\prime}, \sigma K \cap \sigma^{\prime} K \subset$ $\sigma G_{1} \cap \sigma^{\prime} G_{1}=\varnothing$ and also $\sigma^{\prime} K \subset V, \sigma K \subset V$. But by the choice of $K, \mu(K)>0$ and since $\mu$ is left quasi-invariant $\mu(\sigma K)>0$ for every $\sigma$. We deduce that there are uncountably many mutually disjoint compact sets (viz. copies of $K$ ) of $\mu$-measure $>0$ contained in $V$. This implies that $\mu(V)=+\infty$; which contradicts the choice $\mu(V)<+\infty$. Hence $G$ is locally compact, proving the theorem.

We consider below some examples of non-Baire groups relative to the question of the existence of q.-i.R. measures.

EXAMPLE 1. Let $G$ be a countable group and $\tau$ a Hausdorff topology which is not the discrete topology. The additive group of rational numbers $Q$ provides one such example. Also, the additive group of integers can be given a nonmetrizable topology (obviously not locally compact) under which it is a Hausdorff topological group [4, p. 27]. On any such topological group $G$ the following measure $\mu$ is obviously Radon and both left and right quasi-invariant. Suppose $G=\left\{a_{n}\right\}_{1}^{\infty}$ and let $\mu(E)=\sum_{k \in M}\left(1 / 2^{k}\right)$ if $E=\left\{a_{k} \mid k \in M \subset N\right\}$.

EXAMPLE 2. Consider the product group $G \times H$ with the product topology where $G$ is as in Example 1 and $H$ is a locally compact group (and $H$ uncountable). Let $\lambda=\mu \otimes \nu$ where $\mu($ (⿻) is a quasi-invariant measure on $G$ and $v$ a left invariant Haar measure on $H$. It is obvious that $G \times H$ is uncountable and not Baire. The Radon measure $\lambda$ on $G \times H$ is left quasi-invariant in virtue of the following proposition.

Proposition 1. Let $\mu_{i}$ be a left q.-i.R. measure on a group $G_{i}, i=1$ to $n$. Then on the product $G=\prod_{i=1}^{n} G_{i}$, the Radon measure $\mu=\mu_{1} \otimes \mu_{2} \otimes \cdots \otimes$ $\mu_{n}$ is left quasi-invariant.

Proof. It is sufficient to prove the proposition for $n=2$. In the general case the proof follows by induction. Suppose $K$ is a compact set $\subset G_{1} \times G_{2}$ 
such that $\mu(K)=0$. Let $K_{y}$ denote the section of $K$ through $y \in G_{2}$, and $A=\left\{y \in G_{2}: \mu_{1}\left(K_{y}\right)>0\right\}$. By Fubini's theorem $\mu_{2}(A)=0$. Suppose $\left(x_{0}, y_{0}\right) \in$ $G$ and $K^{\prime}=\left(x_{0}, y_{0}\right) K$. We observe that $K_{y_{0} y}^{\prime}=x_{0} K_{y}$ and by the left quasiinvariance of $\mu_{1}$ we get that $\mu_{1}\left(K_{y_{0} y}^{\prime}\right)=0$, for every $y \notin A$. Also $\mu_{2}\left(y_{0} A\right)=0$. Again by Fubini's theorem, we conclude that $\mu\left(K^{\prime}\right)=0$. The proof is complete.

REMARK. The above result does not hold good for infinite products. Consider $G=\boldsymbol{R}^{\boldsymbol{N}}$ which is a Baire group. The product of quasi-invariant measures of total mass 1 on $R$ is not quasi-invariant on $G$ since $G$ is not locally compact.

EXAMPLE 3. On the other hand, not every non-Baire group has a left or right q.-i.R. measure on it. In [2] Feldman, generalizing a result of Sudakov [7], has proved that every metrizable linear space with a nontrivial $\sigma$-finite quasi-invariant Borel measure is necessarily finite dimensional. From this it is possible to get a wide class of topological vector spaces that do not admit any q.-i.R. measure on them. In particular, the following two results could be deduced as consequences of Feldman's result. However, we give below proofs based on Theorem 1.

THEOREM 2. Let $F$ be an infinite dimensional separable Fréchet space. Let $\tau$ be any weaker Hausdorff topology on $F$ such that $(F, \tau)$ is a topological vector space. Then on $(F, \tau)$ considered as an Abelian group, there is no q.-i.R. measure.

Proof. Suppose $\mu$ is a q.-i.R. measure on $(F, \tau)$. By the local finiteness of $\mu$ and the fact that $\tau$ on $F$ is Lindelöf (being weaker than a Lindelöf topology) we deduce that $\mu$ is necessarily $\sigma$-finite. Replacing $\mu$ by an equivalent measure, if necessary, we may assume that $\mu$ is totally finite. Now $F$, a separable Fréchet space, is polish and hence the finite Radon measures (and the Borel sets) are the same for $F$ and $(F, \tau)$ [6, Part II]. Hence, $\mu$ is, in fact, a Radon measure on $F$ and it is quasi-invariant. But $F$ being an infinite dimensional Fréchet space (in particular Baire), this is impossible by Theorem 1 . The proof is complete.

Remark. Suppose $F$ is the strong dual of an infinite dimensional Banach space $E$ and $F$ separable. Then $F$ with weak* topology is $\sigma$ compact (viz. the strong closed balls of radii $n$ centre 0 are weak* compact and form a countable covering) and is hence non-Baire. However, by the above theorem there is no quasi-invariant Radon measure on this space.

EXAMPLE 4. The following is a generalization of a theorem of R. $\mathbf{H}$. Cameron [1]. Let $\mathscr{C}_{0}[0,1]=W$ be the space of real valued continuous functions on $[0,1]$ vanishing at the origin. Let $\mathscr{U}$ (resp. $\tau$ ) be the topology of uniform (resp. pointwise) convergence on $W$. Since $(W, \mathscr{U})$ is polish the Borel $\sigma$-algebra $\mathscr{B}$ is the same for $(W, \tau)$ and $(W, \mathscr{Z})$. This $\sigma$-algebra 
is also the Wiener $\sigma$-algebra on $W$. It is obvious that every set in the Wiener $\sigma$-algebra belongs to $\mathscr{B}$. Conversely, a base for open sets in $\tau$ (viz. sets of the form $\bigcap_{1}^{k}\left\{f \in W: f\left(x_{i}\right) \in V_{i}\right\}$ where $x_{1}, \cdots, x_{k} \in[0,1]$ and $V_{i}$ open sets of $R$ ) is contained in the Wiener $\sigma$-algebra. However, since $(W, \tau)$ is a Lindelöf space, every $\tau$-open set is a countable union of open sets belonging to the above base and hence is in the Wiener $\sigma$ algebra. From this it is easily seen that $\mathscr{B}$ is identical with the Wiener $\sigma$-algebra.

THEOREM 3. There is no $\sigma$-finite measure on $\mathscr{B}$ on the space $W$ which preserves sets of measure zero under translation.

Proof. Suppose there is one such measure $\mu$. We may assume without loss of generality that there is a probability measure $v$ on $\mathscr{B}$ which preserves sets of measure zero under translation. But then looking at $\mathscr{B}$ as the Borel $\sigma$-algebra of $(W, \mathscr{U})$ we conclude that $\nu$ is a Radon measure on $(W, \mathscr{U})$, a polish space. This is impossible in view of the Theorem 1. Hence such a measure cannot exist; this completes the proof.

REMARK. Suppose $\mu$ is a $\sigma$-finite measure on $\mathscr{B}$. Then it follows from the above theorem that $\exists$ at least one $f \in W$ which carries a $\mu^{*}$-measurable set to a non- $\mu^{*}$-measurable set (assuming, of course, that there exists at least one non- $\mu^{*}$-measurable set).

\section{BIBLIOGRAPHY}

1. R. H. Cameron, The translation pathology of Wiener space, Duke Math. J. 21 (1954), 623-627. MR 16, 375.

2. J. Feldman, Nonexistence of quasi-invariant measures on infinite-dimensional linear spaces, Proc. Amer. Math. Soc. 17 (1966), 142-146. MR 32 \#7705.

3. C. Gowrisankaran, Radon measures on groups, Proc. Amer. Math. Soc. 25 (1970), 381-384. MR 41 \#384.

4. E. Hewitt and K. A. Ross, Abstract harmonic analysis. Vol. I. Structure of topological groups. Integration theory, group representations, Die Grundlehren der math. Wissenschaften, Band 115, Academic Press, New York; Springer-Verlag, Berlin, 1963. MR 28 \#158.

5. J. C. Oxtoby, Invariant measures in groups which are not locally compact, Trans. Amer. Math. Soc. 60 (1946), 215-237. MR 8, 253.

6. L. Schwartz, Radon measures on general topological spaces, Tata Institute of Fundamental Research Monographs (to appear).

7. V. N. Sudakov, Linear sets with quasi-invariant measure, Dokl. Akad. Nauk SSSR 127 (1959), 524-525. (Russian) MR 21 \#6412.

Department of Mathematics, McGill University, Montreal, Quebec, Canada 